\title{
P01-192
}

\section{TRYPTOPHAN HYDROXYLASE 2 (TPH2) GENE IN BIPOLAR I DISORDER IN THE ROMANIAN POPULATION}

M. Grigoroiu-Serbanescu ${ }^{1}$, C.C. Diaconu ${ }^{2}$, S. Herms ${ }^{3}$, J. Vollmer ${ }^{3}$, C. Bleotu², M.M. Noethen ${ }^{3,4}$, S. Cichon ${ }^{3}$

${ }^{1}$ Psychiatric Genetics Research Unit, Alexandru Obregia Psychiatric Hospital, ${ }^{2}$ Institute of Virology, Bucharest, Romania, ${ }^{3}$ Department of Genomics, Life \& Brain Center, ${ }^{4}$ Institute of Human Genetics, Rheinische Friedrich-Wilhelms University, Bonn, Germany

Objective: Since the discovery of the tryptophan hydroxylase 2 gene (TPH2) several studies reported association of TPH2 genetic variation with bipolar I disorder (BPI). Our objectives were to replicate in the Romanian population the recently described association of a rare functional SNP (rs17110563) and of a haplotype covering the 5' region of TPH2 with BPI (Cichon et al., 2008) and to investigate the influence of the phenotypic traits age-of-onset, family history and parent-of-origin", defined according to clinical criteria, on the degree of association between TPH2 and BPI.

Method: Sixteen TPH2 SNPs were genotyped in a Romanian sample of 198 BPI patients and 180 controls screened for psychiatric disorders. Statistical analysis of the data was performed with Haploview3.32 and FAMHAP.

Results: The functional SNP rs17110563 (encoding a Pro206Ser substitution) was present in Romanian BPI patients and absent in controls. SNPs located in the 5'-region (rs11178997, rs11178998, rs7954758), significantly associated with BPI in German patients were not associated with BPI in Romanian patients at single-marker level, but gave evidence for association at haplotypic level in a subgroup of patients with paternal transmission of BPI. Evidence for association was identified between haplotypes located in the 3'region of TPH2 and $\mathrm{BPI}$ in the overall sample as well as in the subgroups of familial cases, the subgroup with paternal transmission, and the subgroup with $\mathrm{AO} \leq 25$ years.

Conclusion: Our data provide support for the involvement of TPH2 in the etiology of BPI. Reference: Grigoroiu-Serbanescu M et al, Psychiatric Genetics, 2008. 